\title{
ENCOURAGING ENVIRONMENTALLY FRIENDLY DRIVING THROUGH DRIVER ASSISTANCE: THE ECOMOVE PROJECT
}

\author{
Nicola Fricke \& Caroline Schieß1 \\ Institute of Transportation Systems, German Aerospace Center (DLR) \\ Braunschweig, Germany \\ Email: Nicola.Fricke@dlr.de
}

\begin{abstract}
Summary: A questionnaire study was conducted in order to identify relevant driver motives and assistance options for environmentally-friendly driving. Eighteen participants rated items belonging to the three factors time, environment, and consumption, and subsequently evaluated three types of driver assistance systems. Results concerning the motives showed that older drivers (above the age of 65) focused significantly less on the time motive, whereas high annual mileage drivers tended to focus more on the time motive compared to low annual mileage drivers. Small vehicle drivers emphasized more on the environment motive compared to middle-class/van drivers. In terms of the driver assistance options for eco-friendly driving, a display of the current consumption rate through colorcoding received the highest number of first place rankings in the category intelligent vehicle information systems. A function that automatically shuts off the engine after a certain stopping time, as well as optimal gear choice and time to shift, were ranked high in the category intelligent advanced driver assistance systems. In the category intelligent navigation systems, a traffic- and situational adaptive navigation system was ranked the highest most often. Assessment of drivers' preferences additionally showed that most participants preferred the assistance functions to provide visual information as opposed to direct intervention. The results concerning the driver groups and their underlying driving motives as well as the preference statements will be used to inform the design and development of assistance functions for promoting eco-driving within the European eCo Move project.
\end{abstract}

\section{INTRODUCTION}

Green driving is a topic of much interest. This is due to a heightened social awareness and research agendas focusing on environmentally-friendly behavior in drivers. In the european eCo Move project (Cooperative Mobility Systems and Services for Energy Efficiency) one aspect is encouraging eco-friendly driving through the development of intelligent driver assistance system functions. Such support can be implemented by displaying information on fuel efficient driving, providing feedback on the current driving behavior or by directly intervening to ensure fuel optimized driving (Voort, Dougherty \& van Maarseveen, 2001). Research suggests that such driver assistance systems can promote eco-friendly driving. Manser et al. (2010) investigated two prototype fuel economy driver interface concepts for their ability to improve fuel consumption. One of the concepts displayed fuel economy information whereas the other concept provided information about the current acceleration behavior. In a usability study, participants were asked to drive fuel efficient and results indicated that both of the measures (displays and instruction) led to decreased fuel consumption. Another study found a beneficial effect of a fuel-efficiency 
support tool which provided advice on how to change driving, e.g. to shift to the next gear (Voort et al., 2001). Results of their driving simulator study revealed the largest savings in fuel consumption for the new support tool, as opposed to driving without support, and to an existing system which displays the average consumption. A general overview of currently existing inmarket fuel economy driver interfaces can be found in Jenness et al. (2009). Other research projects aim at developing assistance systems that include several assistance components, e.g. the FootLITE system which gives feedback on the current driving style and pre- and on-trip information about the road network (Fairchild, et al., 2009). All of the reported studies support the hypothesis that driver assistance systems can promote green driving. These studies, however, do not provide detailed information on which system functions might be especially beneficial for certain driver groups. There is evidence that driver characteristics, such as gender, have a differential impact on fuel saving performance when using the same eco-support system (e.g. Gravin, Manser \& Becic, 2010). Other aspects which might lead to varying effects in terms of acceptance and performance might be age, annual mileage, vehicle type and driving motives. This statement is supported by findings that hybrid vehicle drivers tended to rate statements related to different motivators such as "reducing negative environmental impact of driving", "getting the best possible fuel efficiency", and "reducing fuel costs" as being more important compared to normal vehicle drivers (Jenness et al., 2009). Therefore, it seems important to design driver assistance which not only focuses on current driving behavior and fuelconsumption - but potentially provides information on other aspects such as possible time savings or costs. This might help to better meet driver preferences and needs and additionally enhance eco-friendly driving. In order to develop such driver support, one task in the eCo Move project was to identify underlying driving motives and relevant driver groups and explore possible assistance options.

\section{Driving motives}

As a first step, relevant driving motives were identified by conducting a questionnaire study. Thirty-nine items related to eco-friendly driving were used and participants were required to rate the items on a five-point rating scale ( $1=$ "strongly disagree", $5=$ "strongly agree"). Analyses for different driver groups were performed and the following summary of results is based on the answers of $\mathrm{N}=166$ participants. Drivers with a high annual mileage, as well as younger drivers below 25 years of age, showed high scores on items related to a factor which can be interpreted as time. Middle-aged and older drivers as well as small vehicle users and drivers using their car primarily for leisure activities, emphasized more on items which can be summarized by environmental orientation. Female drivers stated a high locus of control for behaving ecofriendly and were found to perceive their car as an object of utility. Older drivers $(\geq 65$ years of age) indicated a higher focus on low fuel consumption than middle aged and young drivers. In addition to these differences in driver groups, participants also mentioned several applications for eco-friendly driving assistance in open-response style questions. Results showed that the most frequently mentioned assistance options were intelligent vehicle information systems (iVIS, $16.5 \%$ ), intelligent-advanced driver assistance systems (iADAS, $16.5 \%$ ) and intelligent traffic management (iTM, $19.8 \%$ ). Intelligent navigation systems (iNavi, $4.1 \%$ ) was mentioned less frequently in general, but was mentioned often by people who indicated using their car for leisure activities (12\% within that group). More details on the study and the results are provided elsewhere (Schieß1 et al., in press). 
Based on this preliminary identification of driver groups and driver support categories, the following HMI-study was designed to further explore driver assistance function alternatives for the identified factors/motives time, environmental orientation and fuel consumption. The aspect "high locus of control" was not a focus in this study because of limited options for developing an HMI related to this aspect.

\section{HMI-DESIGN STUDY}

The aim of the study was gathering more input for the design of appropriate driver support for the identified motives and groups. The study consisted of five parts (please note that not all parts are covered in the results section). In the first part, demographic data were assessed. During the second part of the study, seventeen items from the first questionnaire study, which were associated with the attitudes/motives regarding time, environment, and consumption, were used. These motives were chosen since these can be targeted by a driver support system (e.g. by presenting information related to travel time). An example for an item concerning the motive time would be: "I drive faster than most of the other road users". Participants had to rate these items on a five-point rating scale (1="strongly disagree", 5="strongly agree"). In addition to these items, in the third part of the study, graphical scenarios were used to introduce participants to the subject of eco-friendly driving and related driver assistance systems. For each of the scenarios, participants were required to rate a series of statements on a five-point rating scale.

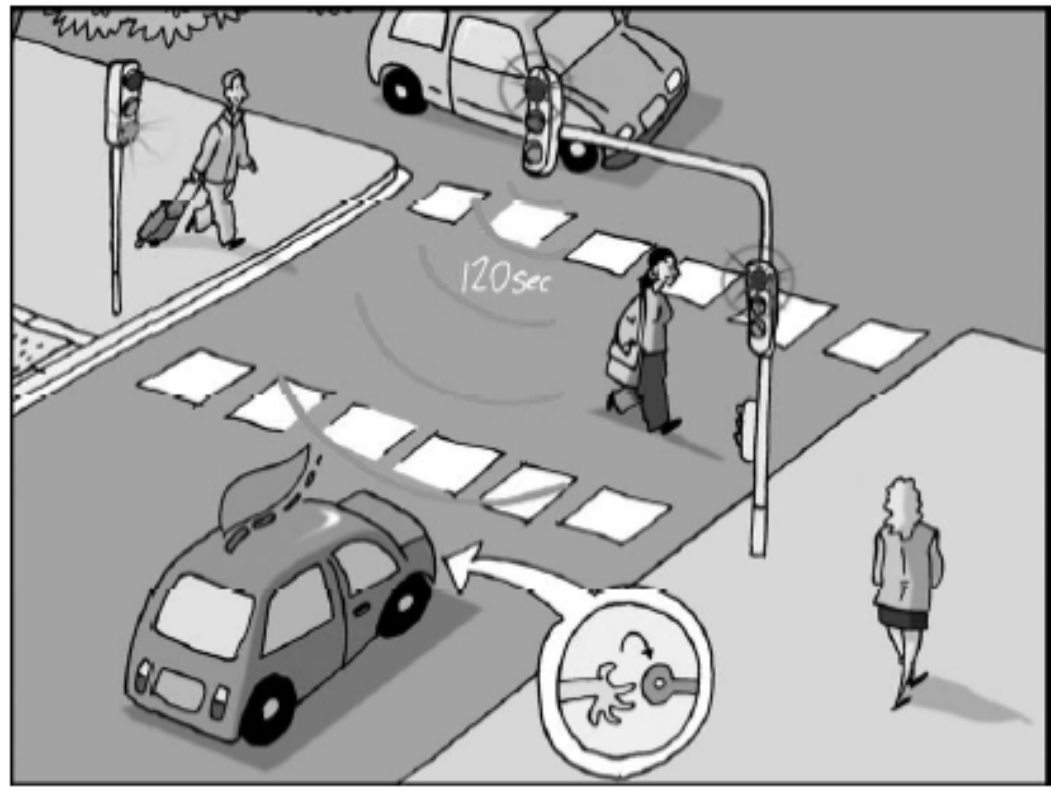

Figure 1. Consumption scenario

One scenario, for instance, was linked to the consumption motive and described a driver assistance system which automatically turns off the engine when the car has stopped for a certain time period (see Figure 1). Afterwards, in part four, participants were asked to name and describe driver assistance functions which they find useful. This was an open-ended question and was queried in an interview-style manner. Moreover, participants were asked to propose specific design implementations. In part five, three relevant driver assistance function groups mentioned earlier from the previous questionnaire were evaluated: iVIS, iADAS and iNavi. Each group was 
introduced to the task by another scenario picture and a table with several design options which the participants had to rate and comment on. One example for an iVIS option was a light display from left to right above the speedometer where fewer lights are associated with less fuel consumption (for all options please refer to Tables 2-4). At the end of the study, participants had to define the three best options for each of the driver assistance function groups and rank them.

\section{Participants and procedure}

The study was conducted at the German Aerospace Center (DLR). One session took about 45-60 minutes, depending on the time spent during the questionnaire and interview phases. Eighteen drivers participated, eight of them were female and the mean age was 41 years (Min: 20 years, Max: 67 years). The mean value for possession of the driving license was 21 years and most drivers belonged to the low and medium annual mileage categories $(9000,9000-20000,>$ $20000 \mathrm{~km} /$ year). Participants received a compensation of $8 €$.

\section{Results of the HMI-study}

Single factor analyses (ANOVAs) or t-tests for independent samples were calculated for the driver groups concerning age, gender, annual mileage, and vehicle models. In the following paragraphs only the significant or tendency effects $(p<.10)$ are reported. Items belonging to each of the factors time, environment, and consumption were summarized by calculating mean values. Higher mean values on these factors refer to a higher focus on this motive.

Results show a tendency effect for the factor time and the driver group annual mileage $(\mathrm{F}=2.713$, $\mathrm{p}=0.099)$. Table 1 shows the descriptive values. The data indicate that high annual mileage drivers tended to rate time as being more important compared to low annual mileage drivers.

Table 1. Descriptive values for the annual mileage group and the motive time

\begin{tabular}{lccccc}
\hline & $\mathrm{N}$ & Mean & SD & Minimum & Maximum \\
\hline$\leq 9000 \mathrm{~km}$ & 7 & 2.80 & 0.25 & 2.54 & 3.31 \\
$9001-20000 \mathrm{~km}$ & 6 & 3.01 & 0.29 & 2.54 & 3.38 \\
$\geq 20000 \mathrm{~km}$ & 5 & 3.14 & 0.20 & 2.92 & 3.38 \\
\hline
\end{tabular}

For the age-groups ( $\leq 24$ years, 25-64 years, $\geq 65$ years) a significant effect was found for the factor time $(\mathrm{F}=4.859, \mathrm{p}<.05)$. Post-hoc comparisons (bonferroni, $\mathrm{p}<.05)$ showed a difference between the young (Mean: 3.17) and older drivers (Mean: 2.74). Older drivers rated time-related items significantly lower, indicating that they focused less on the time motive.

Drivers were also classified based on the vehicles they drove at the time of the study. The frequency of vehicle models in the present study was as follows: small vehicles $(n=9)$, middleclass vehicles $(n=5)$, van $(n=3)$ and a missing value. No other vehicle models were mentioned by the participants. Therefore, participants were categorized based on their vehicle models into "small vehicles" vs. "middle-class/van". A t-test for independent samples showed a significant effect for the motive environment $(\mathrm{t}=2.471, \mathrm{p}<.05)$. Small vehicle drivers rated items related to the environment motive significantly higher (Mean: 2.96) compared to middle-class/van drivers (Mean: 2.17). 


\section{Rankings of the assistance options}

Participants received lists describing assistance options for the three groups: iVIS, iADAS and iNavi. They were required to rank the best three options for each system. Ranks were given multiple times, e.g. some participants gave the third rank to multiple options.

In the category iVIS, the display of the current consumption rate through color-coding received the highest number of first place rankings and also the highest number of total rankings. Two other light displays received three number one rankings, one was a digital light display from green to red, the other one consisted of lights above the speedometer. An auditory/visual consumption display additionally received three number one rankings. A consumption display based on the driving profile received the highest number of rankings for second place and the second highest number of total rankings (11). The full list of rankings is provided in Table 2.

Table 2. Rankings for the iVIS consumption displays

\begin{tabular}{lcccc}
\hline & Ranking 1 & Ranking 2 & Ranking 3 & Total \\
\hline Display of current consumption rate (green, yellow, red) & 8 & 2 & 3 & 13 \\
Digital light display (green to red) & 3 & 4 & 2 & 9 \\
Auditory/visual consumption display & 3 & 2 & 1 & 6 \\
Lights above the speedometer that show the consumption rate & 3 & 1 & 2 & 6 \\
Consumption display based on driving profile & 1 & 6 & 4 & 11 \\
Display of a consumption-diary & 1 & 2 & 4 \\
Display of the monetary savings & 1 & 2 & 0 & 3 \\
Display of consumption savings compared to the last gas tank & 1 & 2 & 0 & 3 \\
Display consumption/time & 0 & 3 & 3 & 6 \\
Display of possible savings based on kilometres & 0 & 3 & 2 & 5 \\
\hline
\end{tabular}

A system that automatically turns off the engine was ranked number one most often in the category iADAS and also received the highest number of overall ratings. Another preferred option was displaying the optimal gear for the current driving situation. Displaying the optimal time for shifting gears received the highest number of second place rankings. The full list of rankings is provided in Table 3 .

Table 3. Rankings for the iADAS

\begin{tabular}{lcccc}
\hline & Ranking 1 & Ranking 2 & Ranking 3 & Total \\
\hline System that automatically turns off the engine & 6 & 4 & 2 & 12 \\
Display of the optimal gear & 4 & 4 & 2 & 10 \\
Information about usage of additional systems & 2 & 2 & 3 & 7 \\
Warning based on the driving behaviour & 2 & 2 & 1 & 5 \\
Motor engine adapting to a driving profile & 2 & 2 & 0 & 4 \\
Display of the optimal time to shift gears & 1 & 5 & 2 & 6 \\
Automatic gear changing based on optimal gear & 1 & 2 & 1 & 4 \\
Comparison of current versus optimal driving style & 1 & 2 & 2 & 4 \\
Display of the current driving style & 0 & 2 & 2 \\
Active gaspedal & 0 & 1 & 3 \\
\hline
\end{tabular}


Traffic- and situational adaptive navigation was ranked highest most often in the category iNavi, followed by route suggestions that are based on possible savings such as time-, consumption- or emission savings. See Table 4 for all rankings.

Table 4. Rankings for iNavi

\begin{tabular}{lcccc}
\hline & Ranking 1 & Ranking 2 & Ranking 3 & Total \\
\hline Traffic- and situational adaptive navigation & 7 & 5 & 4 & 16 \\
Route suggestions based on possible savings & 6 & 7 & 3 & 16 \\
Route planning based on the purpose of the drive & 4 & 1 & 3 & 8 \\
Display of upcoming curves, traffic lights etc. & 3 & 2 & 2 & 7 \\
Route planning based on driving behaviour & 1 & 1 & 4 & 6 \\
Display of the topography of the route & 0 & 1 & 1 & 2 \\
\hline
\end{tabular}

\section{Results of the interviews}

The open ended interview responses were classified into the same three driver support categories. iADAS (89\%) and iVIS (67\%) focusing on displaying fuel consumption were mentioned most often. iNavi was referred to by $44 \%$ of the participants, $39 \%$ requested a system automatically turning off the engine and $28 \%$ favored a post-trip evaluation of their driving behavior. In a second question, participants were asked about the specific implementations of the system, e.g. which modality should be used and what level of support they would prefer. Most participants preferred the system to provide information $(83 \%)$ via the visual modality $(61 \%)$. About 33\% explicitly stated that they do not wish any auditory signals. On the contrary, $28 \%$ of the participants mentioned that they would like to have auditory support and another $28 \%$ preferred interventions by the system. Participants named a few design recommendations such as using visual blinking which increases if consumption is too high, a laughing or crying smiley for post-trip evaluation of driving performance and speech such as "higher gear" or "engine off" for driving advice. One comment suggested using steering wheel vibrations if the driver is speeding.

\section{CONCLUSIONS AND FUTURE WORK}

The aim of this study was to gain more information on which driver groups are associated with certain driving motives, possible eco-driving assistance options, and design implementations. First of all, the results showed some differences in terms of driver groups and motives. Concerning the motive time, young drivers were found to rate the respective items more positively and to focus more on this motive. Moreover, annual mileage drivers showed a tendency to focus on this motive. Small vehicle users were found to focus on the environment motive which is also consistent with the findings from the previously conducted questionnaire study. This means that for younger and high annual mileage drivers one beneficial strategy to enhance environmentally friendly driving could be providing information or assistance for faster travel times. One possibility to do this is by minimizing stops. This strategy has also been shown to lead to higher fuel consumption savings compared to reducing speed and acceleration (Evans, 1979). On the contrary, this information might not be as beneficial for small vehicle users who tended to focus on the environment motive. In this case, the relevant information to display could be the amount of reduced $\mathrm{CO}^{2}$ emissions. These results give some directions for what type of information could be helpful to increase eco-friendly driving and the acceptance of eco- 
assistance systems. The subsequent parts of this study focused on the actual design of support and assistance functions. First of all, auditory warnings are problematic for iVIS or iADAS several comments indicated that visual-only information/warning is preferred. Additionally, optimal gear choice and time to shift as iADAS applications received many first and second place rankings. Generally, speech for information and recommendations was mentioned as preferred compared to tone signals. For iVIS systems, the visual presentation format was often suggested using simple information, for example by displaying colored lights associated with speed.

The results presented in this paper will be used as a basis for the further development of HMI solutions and concepts within the eCo Move project. Further research activities aim at developing general guidelines for the development of HMI strategies, functions and displays for eco-friendly driving. Moreover, experimental driving studies are planned for investigating the interactions between the driver and the support systems and possible side-effects induced by these assistance functions.

\section{ACKNOWLEDGMENTS}

This work is part of the eCo Move project (Cooperative Mobility Systems and Services for Energy Efficiency) funded by the $7^{\text {th }}$ framework program - information society technologies for clean and efficient mobility of the European Commission.

\section{REFERENCES}

Evans, L. (1979). Driver behaviour effects on fuel consumption in urban driving. Human Factors 21 (4), 389-398.

Fairchild R.G., Brake J.F., Thorpe N., Birrell S.A., Young M.S., Felstead T.J., Fowkes M. (2009). Using On-board Driver Feedback Systems to Encourage Safe, Ecological and Efficient Driving: The Foot-LITE Project. Artificial Intelligence \& Simulation of Behaviour Symposium: Persuasive Technology and Digital Behaviour Intervention, Edinburgh, Scotland, April 28-31.

Graving, J. S., Manser, M. P. \& Becic, E. (2010). Reduction in Fuel Consumption Depends on the Fuel Economy Display and Driver Sex: An Observed Interaction. In Adjunct Proceedings of the Second International Conference on Automotive User Interfaces and Interactive Vehicular Applications (AutomotiveUI 2010), November 11-12, 2010, Pittsburgh, Pennsylvania, USA.

Jenness, J. W., Singer, J., Walrath, J. \& Lubar, E. (2009). Fuel Economy Driver Interfaces: Design Range and Driver Opinions. Report on Task 1 and Task 2. National Highway Traffic Safety: Technical Report (DOT HS 811 092).

Manser, M. P., Rakauskas, M., Graving, J. \& Jenness, J. W. (2010). Fuel Economy Driver Interfaces: Develop Interface Recommendation. Report on Task 3. National Highway Traffic Safety: Technical Report (DOT HS 811 319).

Schieß1, C., Fricke, N. \& Staubach, M. (in press). Identification and analysis of motivators for ecofriendly driving within the eCoMove Project. $8^{\text {th }}$ ITS European Congress, June 6-9 $9^{\text {th }} 2011$, Lyon, France.

Voort, M., Dougherty, M. S. \& van Maarseveen, M. (2001). A prototype fuel-efficiency support tool. Transportation Research Part C, 7, 279-296. 